\title{
SETS OF CONSTANT RELATIVE WIDTH AND CONSTANT RELATIVE BRIGHTNESS $\left({ }^{(}\right)$
}

\author{
BY \\ G. D. CHAKERIAN
}

1. Introduction. In "relative differential geometry" one is given a fixed convex body $E$, which serves as a "unit sphere" in the geometry (see [3, p. 64]). The "relative width" of any convex body $K$ is then defined as twice its ordinary width divided by the width of $E$ in the same direction. The "relative brightness" of $K$ is the ordinary brightness divided by the brightness of $E$ in the same direction. We shall be concerned with some properties of sets of constant relative width and sets of constant relative brightness. If $E$ is the ordinary unit ball, then these are the ordinary sets of constant width and constant brightness respectively.

If $E$ is centrally symmetric and $K$ has constant width relative to $E$, then this simply means that $E$ is homothetic to the difference body of $K$. In $\S 6$ we are led to some sharpenings of the inequalities for the volume of the difference body in $E_{2}$ and $E_{3}$. There we also have, incidentally, a generalization and simple proof of the theorem that the insphere and circumsphere of a set of constant width 1 are concentric and their radii sum to 1 (see [3, p. 127]). Properties of sets of constant relative width are studied in [6] and [11].

It is known that if a 3-dimensional convex body has both constant width and constant brightness, then it is a sphere (see [3, p. 140], [13]). The analogue of this in relative geometry is the following. Let $E$ be a 3-dimensional centrally symmetric convex body. Let $K$ be a convex body whose width function is proportional to that of $E$, and whose brightness function is also proportional to that of $E$. Then $K$ is homothetic to $E$. We prove this in $\S 5$, with smoothness restrictions on $K$ and $E$. It would be desirable to remove these restrictions, since the theorem appears to be true without them.

In $\S 2$ we develop some machinery needed in other sections. Much of this is known or is a straightforward generalization of work of Alexandrov [1], and Firey [8]. This material is of interest in its own right, and we give the development with a generality greater than our applications require.

A résumé of relative differential geometry is given in $\$ 4$. This is again in a form more general than our applications require, but the account is, we hope, of independent interest.

I am greatly indebted to Professor W. J. Firey for some stimulating discussions related to material in this paper.

Received by the editors October 3, 1966.

(1) Research partially supported by NSF Grant GP-6230. 
2. The surface area function and mixed volumes. By a "convex body" we shall mean a compact, convex subset of $n$-dimensional Euclidean space $E_{n}$. We shall denote the standard unit ball in $E_{n}$ by $U$, so $U=\left\{x \in E_{n}:|x| \leqq 1\right\}$. Then the boundary of $U$, denoted by $\partial U$, is $\left\{x \in E_{n}:|x|=1\right\}$. We do not index $U$, hoping that its dimension will always be evident from context. If $\lambda$ is a real number, and $S \subset E_{n}$, then $\lambda S=\{\lambda x: x \in S\}$. If $S_{1}, \ldots, S_{r} \subset E_{n}$, then $S_{1}+\cdots+S_{r}=\left\{x_{1}+\cdots+\right.$ $\left.x_{r}: x_{i} \in S_{i}, i=1, \ldots, r\right\} . A, B \subset E_{n}$ are "homothetic" if $A$ is a translate of $\lambda B$, for some $\lambda>0$.

If $K_{1}, \ldots, K_{r}$ are convex bodies in $E_{n}$, and $\lambda_{1}, \ldots, \lambda_{r}$ are nonnegative real numbers, then the volume $V\left(\lambda_{1} K_{1}+\cdots+\lambda_{r} K_{r}\right)$ of the convex body $\lambda_{1} K_{1}+\cdots+\lambda_{r} K_{r}$ is a homogeneous polynomial in $\lambda_{1}, \ldots, \lambda_{r}$ of the form

$$
V\left(\lambda_{1} K_{1}+\cdots+\lambda_{r} K_{r}\right)=\sum \lambda_{i_{1}} \cdots \lambda_{i_{n}} V\left(K_{i_{1}}, \ldots, K_{i_{n}}\right)
$$

where the sum runs over all $n$-tuples of integers $1 \leqq i_{1}, \ldots, i_{n} \leqq r$. The coefficients $V\left(K_{i_{1}}, \ldots, K_{i_{n}}\right)$ of this polynomial are the "mixed volumes," and are symmetric in their arguments. Let $r, s$ be nonnegative integers, with $r+s \leqq n$, and let $C_{1}, \ldots$, $C_{n-r-s}, K_{1}, K_{2}$ be convex bodies in $E_{n}$. For the moment, set

$$
V\left(K_{1}, r ; K_{2}, s ; C\right)=V\left(K_{1}, \ldots, K_{1}, K_{2}, \ldots, K_{2}, C_{1}, \ldots, C_{n-r-s}\right),
$$

where $K_{1}$ appears $r$ times and $K_{2}$ appears $s$ times. Then the Fenchel-Alexandrov inequalities state that

$$
V^{r+s}\left(K_{1}, r ; X_{2}, s ; C\right) \geqq V^{r}\left(K_{1}, r+s ; K_{2}, 0 ; C\right) V^{s}\left(K_{1}, 0, K_{2} ; r+s ; C\right),
$$

(see [4, p. 50]). The following conditions concerning equality in (2.2) will be important [4, p. 51]. A convex body in $E_{n}$ is said to be "regular" if it has nonvoid interior and its boundary is a hypersurface of class $C^{2}$ having everywhere positive curvature. Now suppose $C_{1}, \ldots, C_{n-r-s}$ are regular, and $K_{1}, K_{2}$ each have dimension at least $r+s$. Then if equality holds in (2.2), $K_{1}$ and $K_{2}$ are homothetic. For a detailed account of mixed volumes, see [3], [4].

Let $\mathscr{B}$ be the field of Borel subsets of the unit sphere $\partial U$. Let $K_{1}, \ldots, K_{n-1}$ be convex bodies with nonvoid interior in $E_{n}$. The "mixed surface area function," denoted by $S\left(K_{1}, \ldots, K_{n-1} ; \omega\right)$, is a certain completely additive set function, defined for $\omega \in \mathscr{B}$, having the property that for any convex body $K_{0} \subset E_{n}$,

$$
V\left(K_{0}, K_{1}, \ldots, K_{n-1}\right)=\frac{1}{n} \int_{\partial U} K_{0}(u) S\left(K_{1}, \ldots, K_{n-1} ; d u\right),
$$

where $K_{0}(u)$ is the supporting function of $K_{0}$, and the integral on the right-hand side of (2.3) is the Radon-Stieltjes integral of $K_{0}(u)$ with respect to $S\left(K_{1}, \ldots\right.$, $\left.K_{n-1} ; \omega\right)$. One calls $S(K ; \omega) \equiv S(K, \ldots, K ; \omega)$ the "surface area function" of $K$. For properties of these set functions, see [1], [4], [7].

Let $K_{1}, \ldots, K_{n-1}$ be convex bodies in $E_{n}$, and let $u \in \partial U$. Let $K_{1}^{\prime}, \ldots, K_{n-1}^{\prime}$ be the orthogonal projections of $K_{1}, \ldots, K_{n-1}$ respectively on the $(n-1)$-dimensional 
subspace orthogonal to $u$. Then the "mixed brightness" $\sigma\left(K_{1}, \ldots, K_{n-1} ; u\right)$ of $K_{1}, \ldots, K_{n-1}$ is defined to be the $(n-1)$-dimensional mixed volume $V\left(K_{1}^{\prime}, \ldots, K_{n-1}^{\prime}\right)$. If we let $[u]$ denote the closed segment joining $u$ to the origin, then we have $[3, \mathrm{p}$. 45] $\sigma\left(K_{1}, \ldots, K_{n-1} ; u\right)=V\left(K_{1}^{\prime}, \ldots, K_{n-1}^{\prime}\right)=n V\left(K_{1}, \ldots, K_{n-1}\right.$, [u]). If $K_{1}=K_{2}=\cdots$ $=K_{n-1}=K$, we write $\sigma(K ; u) \equiv \sigma(K, \ldots, K ; u)$, and this is the $(n-1)$-dimensional volume of the orthogonal projection of $K$ onto the subspace orthogonal to $u$. We refer to $\sigma(K ; u)$ as the "brightness" of $K$ in the direction $u$. We have [1, II, p. 1223],

$$
2 \sigma\left(K_{1}, \ldots, K_{n-1} ; v\right)=\int_{\partial U}|(u, v)| S\left(K_{1}, \ldots, K_{n-1} ; d u\right),
$$

where $(u, v)$ is the inner product of $u$ and $v$.

Note that formula (3), page 49, of [3] follows from (2.4), by integrating both sides with respect to $v$ over $\partial U$ and applying (2.3) with $K_{0}=U$.

Using the same kind of convention used in the notation for mixed volumes, in the following we set $S(K, r ; C ; \omega)=S\left(K, \ldots, K, C_{1}, \ldots, C_{n-r-1} ; \omega\right)$, and

$$
\sigma(K, r ; C ; u)=\sigma\left(K, \ldots, K, C_{1}, \ldots, C_{n-r-1} ; u\right),
$$

where $K$ appears $r$ times. Also $S(K, r ; E ; \omega)=S(K, \ldots, K, E, \ldots, E ; \omega)$, and $\sigma(K, r ; E ; u)=\sigma(K, \ldots, K, E, \ldots, E ; u)$, where $K$ appears $r$ times and $E$ appears $n-r-1$ times.

Following an argument of Firey [8, p. 11], we have

THEOREM 1. Let $K_{1}, K_{2}$ be centrally symmetric convex bodies in $E_{n}$, each with dimension $\geqq r$, and let $C_{1}, \ldots, C_{n-r}$ be regular convex bodies. Suppose

$$
\sigma\left(K_{1}, r-1 ; C ; u\right)=\sigma\left(K_{2}, r-1 ; C ; u\right),
$$

for all $u \in \partial U$. Then $K_{1}$ is a translate of $K_{2}$.

Proof. If $K$ is a sufficiently smooth centrally symmetric convex body, then its supporting function $K(u)$ admits the representation (see [3, p. 29])

$$
K(u)=\int_{\partial U}|(u, v)| h(v) d v,
$$

for some continuous function $h$ on $\partial U$. Then

$$
\begin{aligned}
n V\left(K, 1 ; K_{1}, r-1 ; C\right) & =\int_{\partial U} K(u) S\left(K_{1}, r-1 ; C ; d u\right) \\
& =\int_{\partial U}\left\{\int_{\partial U}|(u, v)| S\left(K_{1}, r-1 ; C ; d u\right)\right\} h(v) d v \\
& =2 \int_{\partial U} \sigma\left(K_{1}, r-1 ; C ; v\right) h(v) d v \\
& =2 \int_{\partial U} \sigma\left(K_{2}, r-1 ; C ; v\right) h(v) d v \\
& =n V\left(K, 1 ; K_{2}, r-1 ; C\right) .
\end{aligned}
$$


Thus, for such $K$ we have

$$
V\left(K, 1 ; K_{1}, r-1 ; C\right)=V\left(K, 1 ; K_{2}, r-1 ; C\right) .
$$

Since the mixed volumes are continuous in each argument, (2.7) holds for any centrally symmetric $K$. In particular, letting $K=K_{1}, K_{2}$, we have,

$$
\begin{aligned}
& V\left(K_{1}, r ; K_{2}, 0 ; C\right)=V\left(K_{1}, 1 ; K_{2}, r-1 ; C\right), \\
& V\left(K_{2}, r ; K_{1}, 0 ; C\right)=V\left(K_{2}, 1 ; K_{1}, r-1 ; C\right) .
\end{aligned}
$$

From (2.2) we have

$$
\begin{aligned}
& V^{r}\left(K_{1}, 1 ; K_{2}, r-1 ; C\right) \geqq V^{r-1}\left(K_{2}, r ; K_{1}, 0 ; C\right) V\left(K_{1}, r ; K_{2}, 0 ; C\right), \\
& V^{r}\left(K_{2}, 1 ; K_{1}, r-1 ; C\right) \geqq V^{r-1}\left(K_{1}, r ; K_{2}, 0 ; C\right) V\left(K_{2}, r ; K_{1}, 0 ; C\right) .
\end{aligned}
$$

It follows from (2.8) and (2.9) that equality holds in (2.9), so $K_{1}$ is homothetic to $K_{2}$. Setting $K_{1}=x+\lambda K_{2}$ in (2.8) shows that $\lambda=1$, so $K_{1}$ is in fact a translate of $K_{2}$, as was to be proved.

THEOREM 2. Let $K_{1}$ and $K_{2}$ be convex bodies in $E_{n}$, each with dimension $\geqq r$, and let $C_{1}, \ldots, C_{n-r}$ be convex bodies with nonvoid interior. Suppose that for all $u \in \partial U$,

$$
\sigma\left(K_{1}, r-1 ; C ; u\right)=\sigma\left(K_{2}, r-1 ; C ; u\right) .
$$

Then

$$
\begin{aligned}
& S\left(K_{1}, r-1 ; C ; \omega\right)+S\left(K_{1}, r-1 ; C ;-\omega\right) \\
& \quad=S\left(K_{2}, r-1 ; C ; \omega\right)+S\left(K_{2}, r-1 ; C ;-\omega\right) .
\end{aligned}
$$

Proof. Set

$$
\Phi_{i}(\omega)=S\left(K_{i}, r-1 ; C ; \omega\right)+S\left(K_{i}, r-1 ; C ;-\omega\right),
$$

$i=1,2$, all $\omega \in \mathscr{B}$. Then, by [7, p. 24],

$$
\int_{\partial U} u \Phi_{i}(d u)=0, \quad i=1,2 .
$$

Suppose that the total mass of $\Phi_{i}$ lay on a great $(n-2)$-sphere $\Sigma_{i}$ with pole $u_{i}$, so

$$
\Phi_{i}\left(\Sigma_{i}\right)=\Phi_{i}(\partial U) \text {. }
$$

Then

$$
\begin{aligned}
4 \sigma\left(K_{i}, r-1 ; C ; u_{i}\right) & =\int_{\partial U}\left|\left(u_{i}, v\right)\right| \Phi_{i}(d v) \\
& =\int_{\Sigma_{i}}\left|\left(u_{i}, v\right)\right| \Phi_{i}(d v)=0,
\end{aligned}
$$

so

$$
V\left(K_{i}^{\prime}, \ldots, K_{i}^{\prime}, C_{1}^{\prime}, \ldots, C_{n-r}^{\prime}\right)=0 \text {, }
$$

where $K_{i}^{\prime}, C_{1}^{\prime}, \ldots, C_{n-r}^{\prime}$ are the projections of $K_{i}, C_{1}, \ldots, C_{n-r}$ respectively on the 
(n-1)-dimensional subspace of $E_{n}$ orthogonal to $u_{i}$. But $C_{1}^{\prime}, \ldots, C_{n-r}^{\prime}$ are each $(n-1)$-dimensional, and $K_{i}^{\prime}$ is of dimension at least $r-1$, so the above mixed volume must be $>0\left(\left[3\right.\right.$, p. 41]). This contradiction shows that the total mass of $\Phi_{i}$ does not lie on a great $(n-2)$-sphere. By [7, p. 16], $\Phi_{i}$ is the surface area function of a convex body $M_{i}$ with nonvoid interior in $E_{n}$. Since $\Phi_{i}(\omega)=\Phi_{i}(-\omega), M_{i}$ is centrally symmetric. By (A) and (2.4), $\sigma\left(M_{1} ; u\right)=\sigma\left(M_{2} ; u\right), u \in \partial U$. Then Theorem 1 (with $r=n, M_{i}=K_{i}, i=1,2$ ) implies $M_{1}$ is a translate of $M_{2}$, so $\Phi_{1}(\omega)=\Phi_{2}(\omega), \omega \in \mathscr{B}$, as was to be proved.

COROLlaRY. Let $E$ be a centrally symmetric convex body with nonvoid interior in $E_{n}$. Let $K$ be a convex body of dimension $\geqq r+1$ such that

$$
\sigma(K, r ; E ; u)=\sigma(E ; u), \quad u \in \partial U .
$$

Then

$$
S(K, r ; E ; \omega)+S(K, r, E ;-\omega)=2 S(E ; \omega) .
$$

THEOREM 3. If $K$ and $E$ are convex bodies with nonvoid interior in $E_{n}$, with $K+(-K)=2 E$, then

$$
\sigma(K, 1 ; E ; u)=\sigma(E ; u), \text { for all } u \in \partial U .
$$

Conversely, if $K$ has dimension $\geqq 2$, and $E$ is regular and centrally symmetric with center at the origin in $E_{n}$, then (B) implies that $K+(-K)=2 E$.

Proof. If $K+(-K)=2 E$, then

$$
\begin{aligned}
2 \sigma(E ; u)=\sigma(K+(-K), 1 ; E ; u) & =\sigma(K, 1 ; E ; u)+\sigma(-K, 1 ; E, u) \\
& =2 \sigma(K, 1 ; E ; u)
\end{aligned}
$$

Conversely, if (B) holds and $E=-E$, then $2 \sigma(E ; u)=\sigma(K+(-K), 1 ; E ; u)$ for all $u \in \partial U$. By Theorem 1, with the given conditions on $K$ and $E, K+(-K)=2 E$. This completes the proof.

COROLLARY. Let $E$ be a centrally symmetric convex body with nonvoid interior and center at the origin in $E_{n}$. Let $K$ be a convex body in $E_{n}$. If $K+(-K)=2 E$, then

$$
S(K, 1 ; E ; \omega)+S(K, 1 ; E ;-\omega)=2 S(E ; \omega), \quad \omega \in \mathscr{B} .
$$

Conversely, if $K$ has dimension $\geqq 2$, and $E$ is regular, then (C) implies that

$$
K+(-K)=2 E \text {. }
$$

Proof. If $K+(-K)=2 E$, then (B) of Theorem 3 holds, so (C) follows from Theorem 2. ((C) also follows, more directly, from the linearity of the mixed surface area function in each argument.) Conversely, if (C) is satisfied, then $\sigma(K, 1 ; E ; u)=\sigma(E ; u), u \in \partial U$, so $K+(-K)=2 E$ by Theorem 3 .

RemarK. Let $U$ be the unit ball in $E_{3}$. Let $x_{0},-x_{0}$ be points outside $U$, and let $E$ be the convex hull of $\left\{U, x_{0},-x_{0}\right\}$. Then $E$ is a centrally symmetric "cap-body" 
of $U$. An easy computation shows that $\sigma(U, E ; u) \equiv \sigma(E ; u)$; hence $S(U, E ; \omega)$ $\equiv S(E ; \omega)$, by the corollary to Theorem 2 . However $U+(-U) \neq 2 E$; hence some condition, such as regularity, is needed on $E$ in the second half of Theorem 3 and its corollary.

3. Sets of constant relative width. Let $E$ be a convex body with nonvoid interior in $E_{n}$. Let $E(u)$ denote the supporting function of $E$, and let $B_{1}(u)=E(u)+E(-u)$, $u \in \partial U$, be the "width function" of $E . B_{1}(u)$ is the distance between the supporting hyperplanes of $E$ orthogonal to the direction $u$. If $K$ is any convex body in $E_{n}$, then the "relative width" of $K$ in the direction $u$ is defined to be $2 B_{2}(u) / B_{1}(u)$, where $B_{2}(u)=K(u)+K(-u)$ is the width function of $K . K$ is said to have "constant relative width" if its relative width is constant. If $\alpha>0$, then $K$ has constant relative width $2 \alpha$ if and only if $K+(-K)=\alpha(E+(-E))$. One also says, in this case, that $K$ and $\alpha E$ are "equivalent in width" (see [11]). If $E$ is centrally symmetric, with center at the origin, then $K$ has constant relative width $\alpha>0$ if and only if $K+(-K)=\alpha E$. If $E=U$, this gives the ordinary sets of constant width $\alpha$.

If $E$ is a fixed convex body in $E_{n}$, then we shall define the "relative $r$-girth function" of $K \subset E_{n}$ to be $\sigma(K, r ; E ; u), u \in \partial U$. In case $E=U$, we call this simply the " $r$-girth function."

Theorem 3 implies that if $E$ is regular, then $K$ has constant relative width if and only if its relative 1-girth is proportional to $\sigma(E ; u)$. In case $E=U$, this yields the well-known result: $K \subset E_{n}$ has constant width if and only if it has constant 1-girth. In the case $n=3$, the 1-girth is half the perimeter of the orthogonal projection of $K$ onto a plane orthogonal to $u$-that is, half the "girth" of $K$ in the direction $u$. Hence we have the theorem that $K \subset E_{3}$ has constant width if and only if it has constant girth [8].

Theorem 1 imples that if $E$ is regular, then two centrally symmetric convex bodies having the same relative $r$-girth function are translates of each other. In particular, when $E=U$, one has that the only centrally symmetric sets with constant $r$-girth are spheres, and as a further subcase, the only centrally symmetric sets of constant brightness are spheres $[8$, p. 14].

4. Relative differential geometry. In this section, $E$ will be a regular, centrally symmetric convex body with center at the origin in $E_{n}$. We shall use $E$ as the unit sphere for a relative differential geometry [3, p. 64].

If $S$ is any smooth oriented hypersurface, we have the "Gauss mapping" or "mapping by parallel normals": $S \rightarrow \partial U$, defined by sending $x \in S$ into $u \in \partial U$, where $u$ is the unit normal of $S$ at $x$. If $S_{x}$ is the tangent space of $S$ at $x$, then we denote by $L(S, x)$ the canonical linear mapping of $S_{x}$ into the tangent space of $\partial U$ at $u$, associated with the Gauss mapping (one also can identify $L(S, x)$ with a linear mapping of $S_{x}$ into $S_{x}$ ). Let $E_{e}$ denote the tangent space of $\partial E$ at the point $e$ having the same unit normal as $S$ at $x$. Then we can define the linear mapping $J: S_{x} \rightarrow E_{e}$ by $J=L^{-1}(\partial E, e) L(S, x) . J$ is then, in fact, the canonical linear mapping 
associated with the mapping of $S$ into $\partial E$ by parallel normals. If $S$ is also the boundary of a regular convex body, then $L(S, x)$ is invertible, for each $x \in S$, and $J^{-1}=L^{-1}(S, x) L(\partial E, e)$. In this case we can write $J=J(u)$, without ambiguity, where $u$ is the outward unit normal of $S$ at $x$ and $\partial E$ at $e$. The "relative principal radii of curvature," $\bar{R}_{1}, \ldots, \bar{R}_{n-1}$, of $S$ at $x$, are the reciprocals of the eigenvalues of $J$. We will always use the convention $\bar{R}_{1} \leqq \bar{R}_{2} \leqq \cdots \leqq \bar{R}_{n-1}$. The "relative principal directions" are the eigenvectors of $J$ (see [17], [3, p. 64]).

Following [3, p. 64], let $\left\{\bar{R}_{1} \cdots \bar{R}_{v}\right\}$ denote the $\nu$ th elementary symmetric function of $\bar{R}_{1}, \ldots, \bar{R}_{n-1}$. If $S$ is the boundary of a regular convex body $K$, we let $F_{v}(K, u)$ $=\left\{\bar{R}_{1} \cdots \bar{R}_{v}\right\}$, evaluated at the point $x \in S$ with outward unit normal $u$. Then we have $F_{n-1}(K, u)=\operatorname{det} J^{-1}(u)=[\operatorname{det} L(\partial E, e)][\operatorname{det} L(S, x)]^{-1}=\left[R_{1}^{\prime} \cdots R_{n-1}^{\prime}\right]^{-1}\left[R_{1} \cdots R_{n-1}\right]$ where $R_{1}, \ldots, R_{n-1}$ and $R_{1}^{\prime}, \ldots, R_{n-1}^{\prime}$ are the ordinary principal radii of curvature of $S=\partial K$ and $\partial E$ respectively, evaluated at the points with outer normal $u$. It follows from this that

$$
S(K, \omega)=\int_{\omega} F_{n-1}(K, u) S(E ; d u), \quad \omega \in \mathscr{B} .
$$

When $K$ and $E$ are regular, and $\lambda \geqq 0$, the normal representation of the boundary of $K^{\prime}=K+\lambda E$ is $x^{\prime}(u)=x(u)+\lambda e(u), u \in \partial U$ (see [3, p. 56]). Let $J^{\prime}(u), J(u)$ be the tangent maps (defined above) of $\partial K^{\prime}, \partial K$ respectively. From $d x^{\prime}(u)=d x(u)+\lambda d e(u)$, we obtain $\left(J^{\prime}(u)\right)^{-1}=J^{-1}(u)+\lambda I$, where $I$ is the identity. The relative principal radii of curvature $\bar{R}_{1}^{\prime}(u), \ldots, \bar{R}_{n-1}^{\prime}(u)$ of $\partial K^{\prime}$ are the eigenvalues of $\left(J^{\prime}(u)\right)^{-1}$. Hence the last equation gives $\bar{R}_{i}^{\prime}(u)=\bar{R}_{i}(u)+\lambda, i=1, \ldots, n-1$. Thus we have

$$
F_{n-1}(K+\lambda E, u)=\left(\bar{R}_{1}+\lambda\right) \cdots\left(\bar{R}_{n-1}+\lambda\right)=\sum_{r=0}^{n-1}\left\{\bar{R}_{1} \cdots \bar{R}_{r}\right\} \lambda^{n-r-1} .
$$

Using [7, p. 23], and (4.2), we have

$$
\begin{aligned}
\sum_{r=0}^{n-1}\left(\begin{array}{c}
n-1 \\
r
\end{array}\right) S(K, r ; E ; \omega) \lambda^{n-r-1} & =S(K+\lambda E ; \omega) \\
& =\int_{\omega} F_{n-1}(K+\lambda E, u) S(E ; d u) \\
& =\sum_{r=0}^{n-1} \lambda^{n-r-1} \int_{\omega}\left\{\bar{R}_{1} \cdots \bar{R}_{r}\right\} S(E ; d u) .
\end{aligned}
$$

Equating coefficients yields

$$
\left(\begin{array}{c}
n-1 \\
r
\end{array}\right) S(K, r ; E ; \omega)=\int_{\omega}\left\{\bar{R}_{1} \cdots \bar{R}_{r}\right\} S(E ; d u),
$$

a mild generalization of $[7$, p. 26].

It follows immediately from (4.4) that if $\left\{\bar{R}_{1} \cdots \bar{R}_{r}\right\}$ is constant, for a fixed $r$, $1 \leqq r \leqq n-1$, then $K$ is homothetic to $E$. For, in that case, (4.4) implies

$$
S\left(K^{\prime}, r ; E ; \omega\right) \equiv S(E ; \omega)
$$


where $K^{\prime}$ is homothetic to $K$. Hence, from (2.3), $V\left(M, 1 ; K^{\prime}, r ; E, n-r-1\right)$ $=V(M, E, \ldots, E)$ for all convex bodies $M$. Then, the same sort of argument used in Theorem 1 (from (2.7)) establishes that $K^{\prime}$ is homothetic to $E$; hence $K$ is homothetic to $E$. For an alternate proof, and further references, see [3, p. 118].

Some of the integral formulas of relative differential geometry follow from (4.4). For example, (4.4) implies that if $K_{0}$ is any convex body, then

$$
\begin{aligned}
n\left(\begin{array}{c}
n-1 \\
r
\end{array}\right) V\left(K_{0}, 1 ; K, r ; E, n-r-1\right) & =\left(\begin{array}{c}
n-1 \\
r
\end{array}\right) \int_{\partial U} K_{0}(u) S(K, r ; E ; d u) \\
& =\int_{\partial U} K_{0}(u)\left\{\bar{R}_{1} \cdots \bar{R}_{r}\right\} S(E ; d u) .
\end{aligned}
$$

This contains, as a special case, formulas on page 65 of [3], by taking $K_{0}=K$, or $K_{0}=E$.

As another consequence of (4.4), we have,

THEOREM 4. Let $K$ be regular. Then

$$
S(K, r ; E ; \omega)+S(K, r ; E ;-\omega) \equiv S(E ; \omega),
$$

if and only if

$$
F_{r}(K, u)+F_{r}(K,-u)=\left(\begin{array}{c}
n-1 \\
r
\end{array}\right) \text { for all } u \in \partial U .
$$

Proof. This follows immediately from (4.4) and the uniqueness of the surface area function of $E[7$, p. 12].

The following is a useful generalization of a well-known property of sets of constant width [3, p. 128].

THEOREM 5. Let $K$ be regular, with $K+(-K)=\gamma E$ (so $K$ has constant relative width $\gamma)$. Then the relative principal radii of curvature of $K$ satisfy

$$
\bar{R}_{i}(u)+\bar{R}_{n-i}(-u)=\gamma, \quad u \in \partial U, \quad i=1, \ldots, n-1 .
$$

In particular, $F_{1}(K, u)+F_{1}(K,-u)=(n-1) \gamma, u \in \partial U$.

Proof. If $x=x(u)$ and $e=e(u), u \in \partial U$, are the normal representations of $\partial K$ and $\partial E$ respectively, we have $x(u)-x(-u)=\gamma e(u), u \in \partial U$. Hence, $d x(-u)=d x(u)-$ $\gamma \operatorname{de}(u)=J^{-1}(u) \operatorname{de}(u)-\gamma \operatorname{de}(u)=\left(J^{-1}(u)-\gamma I\right) \operatorname{de}(u)=\left(\gamma I-J^{-1}(u)\right) \operatorname{de}(-u)$, where $I$ is the identity map. Therefore, $J^{-1}(-u)=\gamma I-J^{-1}(u)$. Since the eigenvalues of $J^{-1}(u)$ are $\bar{R}_{1}(u), \ldots, \bar{R}_{n-1}(u)$, and those of $J^{-1}(-u)$ are $\bar{R}_{1}(-u), \ldots, \bar{R}_{n-1}(-u)$, the theorem follows from the last equation (recall the $\bar{R}_{i}(u)$ are ordered by magnitude).

5. Nakajima [13] proved that in $E_{3}$ the only regular convex body of constant width which also has constant brightness is the sphere. In this section we generalize that result with

THEOREM 6. Let $E$ be a centrally symmetric, regular convex body in $E_{3}$, with 
center at the origin. Let $K$ be a regular convex body having constant relative width and constant relative brightness (so $K+(-K)$ is homothetic to $E$ and $\sigma(K ; u)$ is proportional to $\sigma(E ; u))$. Then $K$ is homothetic to $E$.

Proof. We have

(i) $K+(-K)=2 \alpha E$, for some $\alpha>0$, and

(ii) $\sigma(K ; u)=\beta \sigma(E ; u)$, for all $u \in \partial U$, some $\beta>0$.

By the corollary to Theorem 2, and Theorem 4, (ii) implies that

$$
F_{2}(K, u)+F_{2}(K,-u)=2 \beta, \quad u \in \partial U .
$$

Hence, using Theorem 5,

$$
\begin{aligned}
2 \beta=\bar{R}_{1}(u) \bar{R}_{2}(u)+\bar{R}_{1}(-u) \bar{R}_{2}(-u) & =\bar{R}_{1}(u) \bar{R}_{2}(u)+\left(2 \alpha-\bar{R}_{1}(u)\right)\left(2 \alpha-\bar{R}_{2}(u)\right) \\
& =2\left[\left(\bar{R}_{1}(u)-\alpha\right)\left(\bar{R}_{2}(u)-\alpha\right)+\alpha^{2}\right] .
\end{aligned}
$$

The existence of an umbilical point of $\partial K$ (that is, a $\xi$ such that $\bar{R}_{1}(\xi)=\bar{R}_{2}(\xi)$ ) follows as in the ordinary Euclidean case. Choosing $u=\xi$ in (5.2) yields

$$
\beta \geqq \alpha^{2} \text {. }
$$

By Theorem 3, (i) implies

$$
\sigma(K, E ; u)=\alpha \sigma(E ; u), \quad u \in \partial U .
$$

The Minkowski inequality in $E_{2}$ (special case of (2.2) with $r=1, s=1$ ) yields

$$
\sigma(K, E ; u)^{2} \geqq \sigma(K ; u) \sigma(E ; u),
$$

with equality holding only if the orthogonal projections of $K$ and $E$ on a plane orthogonal to $u$ are homothetic. (5.4), (5.3), and (ii), imply that equality holds in (5.5) for all $u \in \partial U$, so all the orthogonal projections of $K$ and $E$ are homothetic. It follows that $K$ and $E$ are homothetic (see [9], [14], [15], [18, p. 257]). This completes the proof.

6. Inequalities for the difference body volume. In this section we give some sharpenings of an inequality for the volume of the difference body of a convex body in $E_{2}$ and $E_{3}$.

Let $K$ be a convex body in $E_{n}$. The difference body measure of symmetry, $\delta_{n}(K)$, is

$$
\delta_{n}(K)=2^{n} V(K) / V(K+(-K))
$$

One has, by the Rogers-Shephard inequality [16],

$$
2^{n}\left(\begin{array}{c}
2 n \\
n
\end{array}\right)^{-1} \leqq \delta_{n}(K) \leqq 1,
$$

with equality on the left only for a simplex, and on the right only if $K$ is centrally symmetric. 
Another interesting measure of symmetry is Minkowski's measure of symmetry, $\lambda_{n}(K)$, defined as follows. For each $x \in K$, let $f(x)=\min _{u \in \partial U} K(u) / K(-u)$, where $K(u)$ is the supporting function of $K$ taken with the origin at $x$. Then

$$
\lambda_{n}=\lambda_{n}(K)=\max _{x \in K} f(x) .
$$

One has $[10$, p. 246]

$$
1 / n \leqq \lambda_{n}(K) \leqq 1,
$$

where equality holds on the right if and only if $K$ is centrally symmetric, and on the left if and only if $K$ is a simplex.

Let $K$ be a convex body with nonvoid interior in $E_{n}$. Choose the origin such that $K(u) \geqq \lambda_{n}(K) K(-u), u \in \partial U$. Then

$$
V(K)=\frac{1}{n} \int_{\partial U} K(u) S(K ; d u) \geqq \lambda_{n} V(-K, K, \ldots, K) .
$$

From (2.1) and (6.4), we have in $E_{3}$,

$$
V(K+(-K))=2 V(K)+6 V(-K, K, K) \leqq\left(2+6 / \lambda_{3}\right) V(K) .
$$

From (6.3) and (6.5), one obtains $V(K+(-K)) \leqq 20 V(K)$, a special case of the left-hand inequality in (6.2). If equality holds here, then (6.5) implies $\lambda_{3} \leqq \frac{1}{3}$, so $K$ is a simplex by the condition for equality in (6.3). The analogue of (6.5) for $E_{2}$, and (6.3), yields $A(K+(-K)) \leqq 6 A(K)$, with equality only for the triangle $(A(K)$ denotes the area of $K$ in $E_{2}$ ). Thus (6.5) and its plane analogue can be considered sharpenings of the inequality (6.2) in the cases $n=2,3$.

If we set

$$
\nu_{3}(K)=4 \lambda_{3}(K) /\left(\lambda_{3}(K)+3\right),
$$

then $\nu_{3}$ is an affine invariant measure of symmetry for convex bodies in $E_{3}$. One has $2 / 5 \leqq \nu_{3}(K) \leqq 1$, with equality on the left-hand side if and only if $K$ is a simplex, and on the right if and only if $K$ is centrally symmetric. Algebraic rearrangement of (6.5) yields the following relation between the measures of symmetry $\delta_{3}$ and $\nu_{3}$ :

$$
\nu_{3}(K) \leqq \delta_{3}(K) .
$$

If $K$ is either centrally symmetric, or a simplex, then equality holds in (6.6). It would be interesting to know precisely for which $K$ equality holds in (6.6).

In $E_{2}$, let $A(K)$ represent the area of $K$. Define

$$
\nu_{2}(K)=2 \lambda_{2}(K) /\left(\lambda_{2}(K)+1\right) .
$$

Then $\nu_{2}$ is an affine invariant measure of symmetry for plane convex bodies, with $\frac{2}{3} \leqq v_{2}(K) \leqq 1$. Equality holds on the left only for triangles, and on the right only for centrally symmetric sets. One has, by arguments similar to the above,

$$
\nu_{2}(K) \leqq \delta_{2}(K) .
$$


Let $E$ be a fixed convex body in $E_{n}$. Then the "relative inradius" of any convex body $K$ is the largest number $r$ such that $K$ contains a translate of $r E$, and the "relative circumradius" is the smallest $R$ such that $K$ is contained in a translate of $R E$. In particular, suppose $E=K+(-K)$, so $K$ is of constant relative width 1 in the relative geometry with $E$ as unit sphere. Let $r$ and $R$ be the relative inradius and relative circumradius respectively of $K$. With an appropriate translation, we may assume $K \subset R E$, so $K(u) \leqq R E(u), u \in \partial U$. It follows that $(1-R) E(u)=E(u)$ $-R E(u) \leqq K(u)+K(-u)-K(u)=K(-u), u \in \partial U$, so $(1-R) E \subset K$. Thus $r \geqq 1-R$. A similar argument shows $R \leqq 1-r$. Thus $r+R=1$. Moreover, it is clear from the argument that the relative insphere and relative circumsphere are concentric; i.e., by translating $K$ appropriately, we have $r E \subset K \subset R E$.

Precisely the same sort of argument which led to (6.4) yields

$$
V(K) \geqq((1-R) / R) V(-K, K, \ldots, K) .
$$

Just as (6.5) followed from (6.4), it follows from (6.8) that

$$
V(K+(-K)) \leqq(2(1+2 R) /(1-R)) V(K), \quad K \subset E_{3},
$$

where $R$ is the relative circumradius of $K$, relative to $K+(-K)$.

The "Jung constant" of $K, J(K)$, is the smallest number $\alpha$ having the following property. Whenever $S$ is such that each pair of its points can be covered by a translate of $K$, then $S$ can be covered by a translate of $\alpha K$. Now let $E=K+(-K)$. Then $2 K$ can be covered by a translate of $J(E) E$. But $2 R E$ is the smallest homothet of $E$ which covers $2 K$; hence, for convex bodies in $E_{n}$, we have

$$
2 R \leqq J(E)=J(K+(-K)) .
$$

This implies, from (6.9), that

$$
V(K+(-K)) \leqq\left(4(1+J(E)) /(2-J(E)) V(K), \quad K \subset E_{3},\right.
$$

where $E=K+(-K)$. Bohnenblust [2] proved that $J(E) \leqq 2 n /(n+1)$, for centrally symmetric convex bodies $E \subset E_{n}, n \geqq 2$. Taking $n=3$ and substituting in (6.11) yields $V(K+(-K)) \leqq 20 V(K)$. Thus (6.11) is another sharpening of the difference body inequality in $E_{3}$. Similar arguments yield, for plane convex bodies,

$$
A(K+(-K)) \leqq\left(4 /(2-J(E)) A(K), \quad K \subset E_{2} .\right.
$$

If $K \subset E_{3}$ is an ordinary set of constant width 1 , then $E=K+(-K)=U$. It is known that $J(U)=(3 / 2)^{1 / 2}$ in $E_{3}$. Substitution of this in $(6.11)$, together with $V(K+(-K))=4 \pi / 3$, yields

$$
V(K) \geqq(\pi / 3)\left(3(6)^{1 / 2}-7\right) \approx .365,
$$

a lower bound for the volume of sets of constant width 1 in $E_{3}$ also found in [5]. 


\section{REFERENCES}

1. A. D. Alexandrov, Zur Theorie der gemischten Volumina von konvexen Körpern, Russian, with German summaries, parts I, II, III, IV.

I. Verällgemeinerung einiger Begriffe der Theorie der konvexen Körper, Mat. Sb. 2 (1937), 947-972.

II. Neue Ungleichungen zwischen den gemischten Volumina und ihre Anwendungen, Mat. Sb. 2 (1937), 1205-1238.

III. Die Erweiterung zweier Lehrsätze Minkowskis über die konvexen Polyeder auf beliebige konvexe Flächen, Mat. Sb. 3 (1938), 27-46.

IV. Die gemischten Diskriminanten und die gemischten Volumina, Mat. Sb. 3(1938), 227-251.

2. F. Bohnenblust, Convex regions and projections in Minkowski spaces, Ann. of Math. 39 (1938), 301-308.

3. T. Bonnensen and W. Fenchel, Theorie der konvexen Körper, Springer, Berlin, 1934.

4. H. Busemann, Convex surfaces, Interscience, New York, 1958.

5. G. D. Chakerian, Sets of constant width, Pacific J. Math. 19 (1966), 13-21.

6. H. G. Eggleston, Sets of constant width in finite dimensional Banach spaces, Israel J. Math. 3 (1965), 163-172.

7. W. Fenchel and B. Jessen, Mengenfunktionen und konvexe Körper, Danske Vid. Selsk. Mat.-Fys. Medd. 16 (1938), 3.

8. W. J. Firey, The brightness of convex bodies, Tech. Rep. No. 19, Oregon State Univ., Eugene, Ore., 1965.

9. H. Groemer, Ein Satz über konvexe Körper und deren Projektionen, Portugal. Math. 21 (1962), 41-43.

10. B. Grünbaum, Measures of symmetry for convex sets, pp. 233-270, Proc. Sympos. Pure Math., Vol. 7, Amer. Math. Soc., Providence, R. I., 1963.

11. P. C. Hammer, Convex curves of constant Minkowski breadth, pp. 291-304, Proc. Sympos. Pure Math., Vol. 7, Amer. Math. Soc., Providence, R. I., 1963.

12. T. Kubota, Ein synthetischer Beweis eines Satzes über Eiflächen, Japan. J. Math. 7 (1930), 171-172.

13. S. Nakajima, Eine charakteristische Eigenschaft der Kugel, Jber. Deutsch. Math.-Verein. 35 (1926), 298-300.

14. — Über homothetische Eiflächen, Japan. J. Math. 7 (1930), 167-169.

15. —_, Eine Kennzeichnung homothetischer Eiflächen, Tôhoku Math. J. 35 (1932), 285-286.

16. C. A. Rogers and G. C. Shephard, The difference body of a convex body, Arch. Math. 8 (1957), 220-233.

17. W. Süss, Zur relativen Differentialgeometrie. I; Über Eilinien und Eifächen in der elementaren und affinen Differentialgeometrie, Japan. J. Math. 4 (1927), 57-75.

18. —_ Zur Minkowskis Theorie von Volumen und Oberfäche, Math. Ann. 101 (1929), 253-260.

UNIVERSITY OF CALIFORNia,

Davis, CALIFornia 